MKPH-T-96-32

\title{
A Dispersion Theoretical Approach to the Threshold Amplitudes of Pion Photoproduction
}

\author{
O. Hanstein, D. Drechsel and L. Tiator \\ Institut für Kernphysik, Universität Mainz, 55099 Mainz, Germany
}

(October 9, 2018)

\begin{abstract}
We give predictions for the partial wave amplitudes of pion photoproduction near threshold by means of dispersion relations at fixed $t$. The free parameters of this approach are determined by a fit to experimental data in the energy range $160 \mathrm{MeV} \leq E_{\gamma} \leq 420 \mathrm{MeV}$. The observables near threshold are found to be rather sensitive to the amplitudes in the resonance region, in particular to the $\Delta(1232)$ and $N^{*}(1440)$. We obtain a good agreement with the existing threshold data for both charged and neutral pion production. Our predictions also agree well with the results of chiral perturbation theory, except for neutral pion production off the neutron.
\end{abstract}

\section{INTRODUCTION}

The photoproduction of pions near threshold has been a topic of considerable experimental and theoretical activities over the past years, ever since the Saclay [1] and Mainz [2] groups showed that the data for the reaction $\gamma p \rightarrow p \pi^{0}$ were at variance with the predictions of a low energy theorem (LET), which was derived in the early 70's by de Baenst [3] and Vainshtein and Zakharov [4]. Being based on fundamental principles like Lorentz, gauge and chiral symmetry, this LET predicted the threshold amplitudes in form of a power 
series in $\mu=m_{\pi} / m_{N}$, with $m_{\pi}$ and $m_{N}$ the masses of pion and nucleon, respectively. The discrepancy between the theoretical expectations and the experimental data was eventually explained by Bernard et al. [5] by an explicit one-loop calculation in (relativistic) ChPT. It was shown that the flaw of the LET was in the assumption that the amplitudes would be analytical functions in $\mu$. In fact, they contain logarithmic terms which cancel in the final result but whose expansion leads to previously unexpected contributions to the power series in $\mu$. In the following years, calculations were considerably refined by introducing the heavy baryon formalism to ChPT, leading to a well-defined expansion in $\mu$, and by including higher orders in the chiral expansion, up to order $p^{4}$ in the $s$-wave multipole $E_{0+}$ and $p^{3}$ in the $p$-wave multipoles [6]. To that order there appear 3 low-energy constants, which have to be fitted to the data or estimated by resonance saturation. While there is some doubt concerning the convergence of the loop expansion for the $s$-waves, the expansion for the $p$-waves seems to converge rather well. Moreover, two combinations of the $p$-waves are free of low energy constants, leading to new LETs to order $p^{3}$. On the experimental side, new precision data have been obtained by both the TAPS collaboration at Mainz [7] and the Sascatoon [8] group. These experiments come closer to threshold than previously possible and clearly show that the strength of the $s$-wave multipole is reduced by nearly a factor 2 as compared to the old LET. The calculations of ChPT describe the data quite nicely. However, there remains the question of the convergence of the loop expansion.

Concerning the production of charged pions, the theoretical description is in much better shape. The dominant contribution near threshold is the Kroll-Ruderman term [9], and corrections up to $O\left(\mu^{3}\right)$ have recently been calculated in ChPT [10]. In that case loop corrections are fairly small, which offers the possibility to determine the pion-nucleon coupling constant $g_{\pi N}$ by new precision experiments.

It is the aim of this letter to investigate the threshold production of neutral and charged pion by means of dispersion relations at fixed $t$. The free parameters of this approach have been fitted to experiments in the energy range from $160 \mathrm{MeV}$ to about $420 \mathrm{MeV}$, i.e. we predict the threshold behaviour on the basis of the data at the higher energies. The paper 
is organized as follows. In section [1], we summarize the kinematics and the observables for pion photoproduction. Some information on dispersion relations at fixed $t$ is given in section III, together with a brief discussion of the assumptions and parameters involved in our calculations. We then discuss the predictions of dispersion theory for threshold pion production in section $1 \nabla$. Finally, we summarize our findings and present some conclusions in section $\mathrm{V}$.

\section{THRESHOLD PION PHOTOPRODUCTION}

We consider the reaction

$$
\gamma(q)+N(p) \rightarrow \pi(k)+N^{\prime}\left(p^{\prime}\right)
$$

The 3-momenta of photon and pion in the $\mathrm{cm}$ frame will be denoted by $\vec{q}$ and $\vec{k}$, respectively. We will use $\Theta_{c m}=\Theta$ to describe the scattering angle, $W$ for the $\mathrm{cm}$ energy of the $\pi N$ system, and $E_{\gamma}$ for the lab energy of the incident photon. In the following we will give the multipole expansion for the pertinent observables taking into account only the $s$-wave multipoles $E_{0+}$ and the $3 p$-waves $E_{1+}, M_{1+}$ and $M_{1-}$. We note, however, that our numerical results in section $[\mathrm{IV}$ include the higher partial waves as well. For convenience we also introduce the combinations

$$
P_{1}=3 E_{1+}+M_{1+}-M_{1-}, \quad P_{2}=3 E_{1+}-M_{1+}+M_{1-}, \quad P_{3}=2 M_{1+}+M_{1-} .
$$

With these definitions and approximations, the differential cross section in the $\mathrm{cm}$ frame reads 11

$$
\frac{d \sigma}{d \Omega}=\frac{|\vec{k}|}{|\vec{q}|}\left(A+B \cos \theta+C \cos ^{2} \theta\right),
$$

where

$$
\begin{aligned}
& A=\left|E_{0+}\right|^{2}+\frac{1}{2}\left|P_{2}\right|^{2}+\frac{1}{2}\left|P_{3}\right|^{2}, \\
& B=2 \operatorname{Re}\left(E_{0+} P_{1}^{*}\right), \\
& C=\left|P_{1}\right|^{2}-\frac{1}{2}\left|P_{2}\right|^{2}-\frac{1}{2}\left|P_{3}\right|^{2},
\end{aligned}
$$


leading to the total cross section

$$
\sigma_{\text {tot }}=4 \pi \frac{|\vec{k}|}{|\vec{q}|}\left(\left|E_{0+}\right|^{2}+\left|M_{1-}\right|^{2}+6\left|E_{1+}\right|^{2}+2\left|M_{1+}\right|^{2}\right) .
$$

The single-polarization observables are the beam asymmetry,

$$
\Sigma=\Gamma \sin \theta\left(\left|P_{3}\right|^{2}-\left|P_{2}\right|^{2}\right),
$$

the target asymmetry

$$
T=2 \Gamma \operatorname{Im}\left(\left(E_{0+}+\cos \theta P_{1}\right)\left(P_{3}-P_{2}\right)^{*}\right),
$$

and the recoil polarization

$$
P=2 \Gamma \operatorname{Im}\left(\left(E_{0+}+\cos \theta P_{1}\right)\left(P_{3}+P_{2}\right)^{*}\right),
$$

where

$$
\Gamma=\frac{|\vec{k}| \sin \theta}{2|\vec{q}|}\left(\frac{d \sigma}{d \Omega}\right)^{-1}
$$

The isospin decomposition of the physical amplitudes $\mathcal{M}_{l}(N \pi)$ is given by the linear combinations

$$
\begin{aligned}
& \mathcal{M}_{l}\left(n \pi^{+}\right)=\sqrt{2}\left(\mathcal{M}_{l}^{(0)}+\mathcal{M}_{l}^{(-)}\right)=\sqrt{2}\left(\mathcal{M}_{l}^{(0)}+\frac{1}{3} \mathcal{M}_{l}^{\left(\frac{1}{2}\right)}-\frac{1}{3} \mathcal{M}_{l}^{\left(\frac{3}{2}\right)}\right) \\
& \mathcal{M}_{l}\left(p \pi^{-}\right)=\sqrt{2}\left(\mathcal{M}_{l}^{(0)}-\mathcal{M}_{l}^{(-)}\right)=\sqrt{2}\left(\mathcal{M}_{l}^{(0)}-\frac{1}{3} \mathcal{M}_{l}^{\left(\frac{1}{2}\right)}+\frac{1}{3} \mathcal{M}_{l}^{\left(\frac{3}{2}\right)}\right) \\
& \mathcal{M}_{l}\left(p \pi^{0}\right)=\mathcal{M}_{l}^{(0)}+\mathcal{M}_{l}^{(+)}=\mathcal{M}_{l}^{(0)}+\frac{1}{3} \mathcal{M}_{l}^{\left(\frac{1}{2}\right)}+\frac{2}{3} \mathcal{M}_{l}^{\left(\frac{3}{2}\right)} \\
& \mathcal{M}_{l}\left(n \pi^{0}\right)=-\mathcal{M}_{l}^{(0)}+\mathcal{M}_{l}^{(+)}=-\mathcal{M}_{l}^{(0)}+\frac{1}{3} \mathcal{M}_{l}^{\left(\frac{1}{2}\right)}+\frac{2}{3} \mathcal{M}_{l}^{\left(\frac{3}{2}\right)}
\end{aligned}
$$

\section{DISPERSION RELATIONS AT FIXED $t$}

Assuming analyticity and unitarity, the invariant amplitudes $A(s, t)$ for pion photoproduction may be written as the sum of pole contributions and dispersion integrals. Since 
the Mandelstam variable $t$ is kept fixed, the integral runs over the energy variable $s$ from $\pi N$ threshold to infinity. Though it is generally difficult to prove the validity of dispersion relations, Oehme and Taylor [12 have given such a proof in this case, at least for sufficiently small values of $t$. As usual in this context, the pole terms are given by the Born terms in pseudoscalar pion-nucleon coupling. This may seem to be at variance with the wellestablished fact that pseudovector coupling should be preferred because of chiral symmetry. However, both approaches lead to the same contributions at the pole, and the violation of chiral symmetry by using the pseudoscalar coupling for the Born terms will be removed by appropriate contributions of the dispersion integral.

Starting from these fixed- $t$ dispersion relations for the invariant amplitudes of pion photoproduction, the projection of the multipole amplitudes leads to a well known system of integral equations [13,

$$
\operatorname{Re} \mathcal{M}_{l}(W)=\mathcal{M}_{l}^{\mathrm{P}}(W)+\frac{1}{\pi} \sum_{l^{\prime}} \mathcal{P} \int_{W_{\mathrm{thr}}}^{\infty} K_{l l^{\prime}}\left(W, W^{\prime}\right) \operatorname{Im} \mathcal{M}_{l^{\prime}}\left(W^{\prime}\right) d W^{\prime}
$$

where $\mathcal{M}_{l}$ stands for any of the multipoles $E_{l \pm}, M_{l \pm}$, and $\mathcal{M}_{l}^{\mathrm{P}}$ for the corresponding (nucleon) pole term. The kernels $K_{l l^{\prime}}$ are known, and the real and imaginary parts of the amplitudes are related by unitarity. In the energy region below two-pion threshold, unitarity is expressed by the final state theorem of Watson [14],

$$
\mathcal{M}_{l}^{I}(W)=\left|\mathcal{M}_{l}^{I}(W)\right| e^{i\left(\delta_{l}^{I}(W)+n \pi\right)},
$$

where $\delta_{l}^{I}$ is the corresponding $\pi N$ phase shift and $n$ an integer.

For completeness we note that Eq. (12) assumes that the Compton phase may be neglected, which could introduce systematical errors at the level of one percent. The further numerical solution of Eqs. (11) with the constraints imposed by unitarity follows the method of Schwela et al. [13]. In addition we have taken account of the coupling to higher states neglected in that earlier reference. At the energies above two-pion threshold up to $W=2$ GeV, Eq. (12) has been replaced by an ansatz based on unitarity [13]. Though this ansatz is by no means unique, it was motivated by a comparison with the existing data at the 
higher energies. Furthermore, we assume that the contribution of the dispersive integrals from $2 \mathrm{GeV}$ to infinity is largely dominated by $t$-channel exchange. In earlier references [15] the high-energy behavior has been simulated by contributions of Regge trajectories. This involved a large number of free parameters which had to be fitted to the existing data with large error bars. Since we will restrict our calculations to energies $E_{\gamma} \leq 450 \mathrm{MeV}$, the main $t$-channel contribution should be due to (a fraction of) $\rho$ - and $\omega$-exchange, described by four coupling parameters. Furthermore, we have to allow for the addition of solutions of the homogeneous equations to the coupled system of Eq. (11). The whole procedure introduces 10 free parameters, which have to be determined by a fit to the data. In our data base we have included the recent MAMI experiments for $\pi^{\circ}$ and $\pi^{+}$production off the proton in the energy range from $160 \mathrm{MeV}$ to $420 \mathrm{MeV}$ [7,16,17, both older and more recent data from Bonn for $\pi^{+}$production off the proton [18 20], and older Frascati [21] and more recent TRIUMF data 22] on $\pi^{-}$production off the neutron.

In cases of weak coupling between the respective channels we neglected some of the integral kernels $K_{l l^{\prime}}$ in our fitting procedure. Therefore we iterated the full integral equations (11) to ensure consistency of the method. At low energies the only cases where we found a significant discrepancy between our original solution and the result after iteration, were the $E_{0+}$ amplitudes for neutral pion production. For this there are several reasons. First of all we expect strong effects from isospin breaking and unitarity for the $s$-wave amplitude near threshold. In our approach the assumption of isospin symmetry is quite essential when we impose the phases of the partial waves according to Eq. (12). However, We introduce some isospin breaking by hand via the mass difference of charged and neutral pions. Second, the $E_{0+}$ amplitudes of neutral pion production are sums of large contributions with strong cancelations (see Tab. (I) between the large (pseudoscalar!) Born term and the dispersive integrals. As a consequence slight variations of these contributions can strongly affect the final results. 


\section{RESULTS}

As can be seen from Tab. [ the the $s$-waves of charged pion production at threshold are mainly given by the pole term contributions, while there are only small corrections from the dispersion integrals. In particular there is a strong cancelation between the (large) $P_{33}$ contributions to the $I=\frac{1}{2}$ and $I=\frac{3}{2}$ components entering into the linear combination $E_{0+}^{(-)}$ (see Eqs. (10)). In 23] this contribution had been determined to be $0.05 \times 10^{-3} / m_{\pi}$, assuming only the $M_{1+}\left(\frac{3}{2}\right)$ amplitude to contribute. According to our result this contribution is $0.09 \times 10^{-3} / m_{\pi}$. More interesting is the fact, that the contribution of the $E_{1+}\left(\frac{3}{2}\right)$ multipole which was neglected in [23] is much larger $\left(0.27 \times 10^{-3} / m_{\pi}\right)$. We also stress that the $\Delta$ contribution determined in this way differs even in sign from the corresponding value of $-0.57 \times 10^{-3} / m_{\pi}$ which has been derived in heavy baryon chiral perturbation theory (HBChPT) [10]. However, such differences in individual terms are not unexpected, because there is no unique correspondence between the graphology of perturbation and dispersion theory.

The first prediction for the threshold values of charged pion production was a LET stating that the $s$-wave multipoles are given by the Kroll-Ruderman term $[9], E_{0+}^{\mathrm{thr}}\left(\pi^{+} n\right)=$ $27.6 \times 10^{-3} / m_{\pi^{+}}, E_{0+}^{\mathrm{thr}}\left(\pi^{-} p\right)=-31.7 \times 10^{-3} / m_{\pi^{+}}$, where we assumed $g_{\pi N}=13.4$. Recently, corrections to these values have been calculated in HBChPT, to $O\left(\mu^{2}\right)$. As can be seen from Tab. II there is good agreement for $\pi^{+}$production between our result and ChPT, and both agree nicely with the experiment (see also Fig. 1). In the case of $\pi^{-}$production we find agreement of our result with the old LET value and the result of an old experimental analysis, while ChPT indicates a slightly stronger $E_{0+}$ multipole (see Tab. 【I). The preliminary analysis of the angular distribution in a recent experiment from TRIUMF (experiment E643), in which the inverse reaction $\pi^{-} p \rightarrow \gamma n$ has been studied, resulted in the much larger value $(-34.6 \pm 1.0) \times 10^{-3} / m_{\pi^{+}}$[24]. At variance with that finding, the threshold extrapolation of the total cross sections determined from the same experiment led to $(-32.8 \pm 0.7) \times 10^{-3} / m_{\pi^{+}}$, reasonably close to the theoretical expectations. As has been shown in Fig. 1, our analysis 
describes the angular distribution at two energies quite well, although our solution for the $E_{0+}$ amplitude is significantly smaller than the values according to the analysis of Ref. 24. We note that these threshold data were not included in our fit, and conclude that there must be some inconsistency in the data analysis, probably due to an underestimate of the statistical errors in the angular distributions. There is special theoretical interest in this amplitude because it allows for an independent determination of the difference $a_{1}-a_{3}$ of the pion-nucleon scattering lengths via the Panofsky ratio, $P=\sigma\left(\pi^{-} p \rightarrow \pi^{0} n\right) / \sigma\left(\pi^{-} p \rightarrow \gamma n\right)$. This ratio is well determined by experiment, $P=1.543 \pm 0.008$ [25], and related with the scattering lengths by time reversal,

$$
\left(a_{1}-a_{3}\right)^{2}=9 \frac{q}{k_{0}}\left|E_{0+}^{\mathrm{thr}}\left(\pi^{-} p\right)\right|^{2} P
$$

where $q=|\vec{q}|$ and $k_{0}=\left|\overrightarrow{k_{\pi^{0}}}\right|$ are the $\mathrm{cm}$ momenta of photon and neutral pion at $p \pi^{-}$ threshold. Using $9 q / k_{0}=41.3$, our value of the threshold amplitude, and the measured Panofsky ratio, we find $a_{1}-a_{3}=0.253 / m_{\pi}$. This has to be compared with the value $0.274 \pm$ $0.012 / m_{\pi}$ resulting from a partial wave analysis of pion-nucleon scattering [26] (solution KH80). Recently, $a_{1}-a_{3}$ has also been determined by studying the level spacing of pionic atoms, with a preliminary value of $0.288 / m_{\pi}$ [27].

As has been previously mentioned, an exact prediction of the $s$-wave multipoles for neutral pion production is difficult. The experimental values show a pronounced cusp effect at the $\pi^{+}$threshold, and the expansion in $\mu$ in HBChPT seems to converge only slowly. In the framework of dispersion relations, we observe a delicate cancelation between the large negative value of the (pseudoscalar) Born term and the contributions of the dispersion integrals (see line 3 of Tab.피). Our final threshold value of -1.22 (here and in the following in units of $10^{-3} / m_{\pi}$ ) is obtained after iterating the integral equations, i.e. by inserting the imaginary parts of our best fit into the integrals on the rhs of Eqs. (11). As has been mentioned, the solutions of the best fit have been obtained by neglecting some of the weak couplings in the system of coupled equations. In general, the iterated solutions agreed with the original ones at the per cent level, thus demonstrating the validity of our approximations. 
The only exception to this is the $E_{0+}$ amplitude for neutral pion production, where we obtain about -0.9 at threshold without the iteration [28]. As is shown in Fig. 2, the iterated solution describes the energy dependence of the data very well, in particular the steep rise of $\operatorname{Im} E_{0+}$ at $\pi^{+}$threshold gives rise to the observed cusp effect.

In the case of the reaction $\gamma n \rightarrow n \pi^{0}$, we predict a similarly strong cancelation of Born terms (-5.2) and resonance contributions, leading to a final result of +1.19 (see Tab. 看). However, our result is considerably lower than the value of 2.1 predicted by ChPT. Tab. 目 also shows the influence of the Roper multipole $M_{1-}$ on the dispersion integrals. Its size and even the change of sign for $p \pi^{0}$ vs. $n \pi^{0}$ is of great importance for our final result. As the present data for pion production from the neutron suffer considerably from systematical and statistical errors, the predictive power of our calculation is of course much weaker in this case than for the proton.

In contrast to the $s$-wave amplitudes, the $p$-waves seem to converge much faster in HBChPT [8]. In particular, it has been possible to derive LETs for the combination $P_{1}$ and $P_{2}$ of Eqs. (2). Our results for these multipoles are compared to the predictions of ChPT and experimental analyses in Tab. [1]. There is general agreement, typically within 5\%. The most problematic case is again the multipole $M_{1-}$. As can be seen from Eqs. (6 8), its influence is particularly strong in the case of polarization observables. We have demonstrated this in Figs. 3 and 1 by arbitrarily reducing the strength of this multipole by 50\%, which changes even the sign of the beam asymmetry $\Sigma$.

\section{CONCLUSION}

In conclusion we find a good overall description of the existing threshold data, showing the internal consistency of the data at different energies. However, there also remain some open questions, particularly regarding the size of the multipole $M_{1^{-}}$connected with the Roper resonance, the phases of the photoproduction multipoles at energies above the second

resonance region, and the high-energy behaviour of the amplitudes. There is also some 
doubt concerning the accuracy of the neutron data, and it is quite clear that the present predictions of dispersion theory cannot be better than the data serving as input. The present uncertainties concerning the size of the multipole $M_{1-}$ should be removed by the analysis of the recently measured beam asymmetry $\Sigma$ for neutral pion production off the proton [29]. There is also hope that the proposed experiment $d\left(e, e^{\prime} d\right) \pi^{0}$ [30] will shed some light on the threshold amplitude for neutral pion photoproduction off the neutron. This reaction will be an extremely sensitive test of model predictions and of the size of isospin symmetry breaking in threshold pion production.

\section{ACKNOWLEDGMENTS}

We would like to thank Prof. G. Höhler for very fruitful discussions and the members of the A2 collaboration at Mainz for providing us with their preliminary data, in particular R. Beck, F. Härter, H.-P. Krahn and H. Ströher. This work was supported by the Deutsche Forschungsgemeinschaft (SFB 201). 


\section{REFERENCES}

[1] E. Mazzucato et al., Phys. Rev. Lett. 57 (1986) 3144.

[2] R. Beck et al., Phys. Rev. Lett. 65 (1990) 1841.

[3] P. de Baenst, Nucl. Phys. B 24 (1970) 633.

[4] I. A. Vainshtein and V. I. Zakharov, Nucl. Phys. B36 (1972) 589.

[5] V. Bernard, J. Gasser, N. Kaiser and U.-G. Meißner, Phys. Lett. B 268 (1991) 219.

[6] V. Bernard, N. Kaiser and U.-G. Meißner, Phys. Lett. B 378 (1996) 337,

N. Kaiser, Progr. Part. Nucl. Phys. 36 (1996) 119.

[7] M. Fuchs et al., Phys. Lett. B 368 (1996) 20,

A. Bernstein et al., Phys. Rev. C (1997) in print, preprint nucl-ex/9610005.

[8] J. C. Bergstrom et al., Phys. Rev. C 53 (1996) 1052.

[9] N. M. Kroll and M. A. Ruderman, Phys. Rev. 93 (1954) 233.

[10] V. Bernard, N. Kaiser and U.-G. Meißner, Phys. Lett. B 383 (1996) 116.

[11] D. Drechsel and L. Tiator, J. Phys. G: Nucl. Part. Phys. 18 (1992) 449.

[12] R. Oehme and J. G. Taylor, Phys. Rev. 113 (1959) 371.

[13] D. Schwela and R. Weizel, Z. Physik 221 (1969) 71.

[14] K. M. Watson, Phys. Rev. 95 (1954) 228.

[15] B. H. Kellett, Nucl. Phys. B 25 (1970) 205.

[16] H.-P. Krahn, PhD. thesis, Mainz (1996).

[17] F. Härter, PhD. thesis, Mainz (1996).

[18] D. Menze, W. Pfeil and R. Wilcke, Compilation of pion photoproduction data, Bonn (1977). 
[19] K. Buechler et al., Nucl. Phys. A 570 (1994) 580.

[20] H. Dutz, PhD. thesis, Bonn (1993),

D. Krämer, PhD. thesis, Bonn (1993),

B. Zucht, PhD. thesis, Bonn (1995).

[21] F. Carbonara et al., Nuovo Cim. 13 A (1973) 59.

[22] A. Bagheri et al., Phys. Rev. C 38 (1988) 875.

[23] G. Höhler and W. Schmidt, Ann. Phys. 28 (1964) 34.

[24] Kailin Liu, "Radiative $\pi^{-} p$ capture and the low energy theorem", Ph.D. thesis, University of Kentucky (1994).

[25] J. Spuller et al., Phys. Lett. 67 B (1977) 479.

[26] G. Höhler, Pion-Nucleon Scattering, Landoldt-Börnstein, vol. I/9b2, ed. H. Schopper, Springer (1983).

[27] D. Sigg et al., Phys. Rev. Lett. 75 (1995) 3245.

[28] O. Hanstein, L. Tiator and D. Drechsel, $\pi N$ Newsletter 10 (1995) 144.

[29] R. Beck et al., MAMI Proposal A2/7-94.

[30] A. M. Bernstein, H. Merkel (spokesmen) et al., MAMI Proposal A1/1-96.

[31] V. Bernard, N. Kaiser and U.-G. Meißner, Z. Phys. C 70 (1996) 483.

[32] M. I. Adamovich et al., Sov. J. Nucl. Phys. 7 (1970) 360. 


\section{TABLES}

TABLE I. Decomposition of the $E_{0+}$ amplitudes in units of $10^{-3} / m_{\pi^{+}}$. We give the contributions of the pole terms and of the dispersion integrals (11) over the respective multipoles.

\begin{tabular}{lccccccr}
\hline \hline & pole & $M_{1+}^{\left(\frac{3}{2}\right)}$ & $E_{1+}^{\left(\frac{3}{2}\right)}$ & $E_{0+}$ & $M_{1-}$ & others & sum \\
\hline$E_{0+}\left(n \pi^{+}\right)$ & 26.84 & 0.13 & 0.40 & -0.21 & 0.91 & -0.065 & 27.99 \\
$E_{0+}\left(p \pi^{-}\right)$ & -30.43 & -0.13 & -0.40 & -1.84 & 0.86 & 0.26 & -31.67 \\
$E_{0+}\left(p \pi^{0}\right)$ & -7.63 & 4.15 & -0.41 & 2.32 & 0.29 & 0.068 & -1.22 \\
$E_{0+}\left(n \pi^{0}\right)$ & -5.23 & 4.15 & -0.41 & 3.68 & -0.93 & -0.05 & 1.19 \\
\hline \hline
\end{tabular}

TABLE II. The $E_{0+}$ amplitudes for charged pion photoproduction at threshold in units of $10^{-3} / m_{\pi^{+}}$. Our values are compared with results from chiral perturbation theory [10] and data analysis [32]. We also give our predictions for the reduced $p$-wave multipoles in units of $|\vec{k}||\vec{q}| 10^{-3} / m_{\pi^{+}}^{3}$.

\begin{tabular}{|c|c|c|c|c|c|c|c|c|}
\hline & $\gamma p \rightarrow \pi^{+} n$ & & & & $\gamma n \rightarrow \pi^{-} p$ & & & \\
\hline & $E_{0+}$ & $m_{1-}$ & $e_{1+}$ & $m_{1+}$ & $E_{0+}$ & $m_{1-}$ & $e_{1+}$ & $m_{1+}$ \\
\hline disp. & 28.0 & 6.1 & 4.9 & -9.6 & -31.7 & -8.3 & -4.9 & 11.2 \\
\hline Ref. 110 & $28.2 \pm 0.6$ & & & & $-32.7 \pm 0.6$ & & & \\
\hline Ref. 32 & $28.3 \pm 0.04$ & & & & $-31.8 \pm 0.20$ & & & \\
\hline
\end{tabular}


TABLE III. $s$ - and $p$-wave multipoles of neutral pion photoproduction at threshold in comparison with predictions from ChPT [6, 31] and results of data analysis [7,8]. The unit of the $s$-waves is $10^{-3} / m_{\pi^{+}}$, the unit of the reduced $p$-waves is $|\vec{k} \| \vec{q}| 10^{-3} / m_{\pi^{+}}^{3}$.

\begin{tabular}{|c|c|c|c|c|}
\hline & disp. & ChPT, Refs. [6, 31] & Exp., Ref. [7] & Exp., Ref. [8] \\
\hline$E_{0+}\left(p \pi^{0}\right)$ & -1.22 & -1.16 & $-1.31 \pm 0.08$ & $-1.32 \pm 0.11$ \\
\hline$m_{1-}\left(p \pi^{0}\right)$ & -3.91 & -3.21 & & $-3.38 \pm 0.26$ \\
\hline$e_{1+}\left(p \pi^{0}\right)$ & -0.15 & -0.11 & & $-0.67 \pm 0.15$ \\
\hline$m_{1+}\left(p \pi^{0}\right)$ & 7.07 & 7.45 & & $7.44 \pm 0.04$ \\
\hline$\left(m_{1+}-m_{1-}\right)\left(p \pi^{0}\right)$ & 10.99 & 10.65 & & $10.82 \pm 0.26$ \\
\hline$p_{1}\left(p \pi^{0}\right)$ & 10.52 & 10.33 & $10.02 \pm 0.15$ & $9.2 \pm 0.3$ \\
\hline$E_{0+}\left(n \pi^{0}\right)$ & 1.19 & 2.13 & & \\
\hline$p_{1}\left(n \pi^{0}\right)$ & 7.77 & 7.40 & & \\
\hline$p_{2}\left(n \pi^{0}\right)$ & -8.77 & -8.36 & & \\
\hline
\end{tabular}




\section{FIGURES}
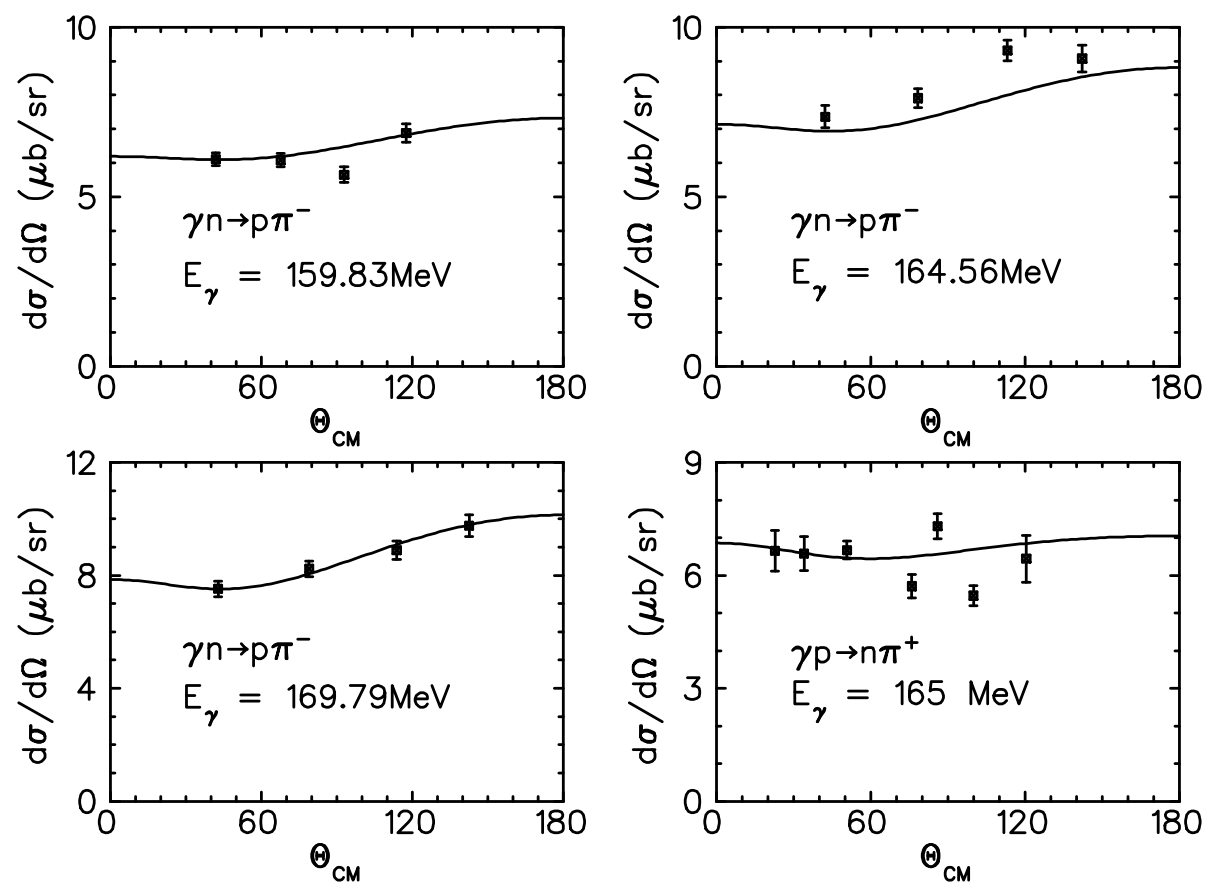

FIG. 1. Differential cross sections for charged pion production. The data for $\gamma n \rightarrow \pi^{-} p$ are taken from Ref. [24], the data for $\gamma p \rightarrow \pi^{+} n$ from Ref. [18].

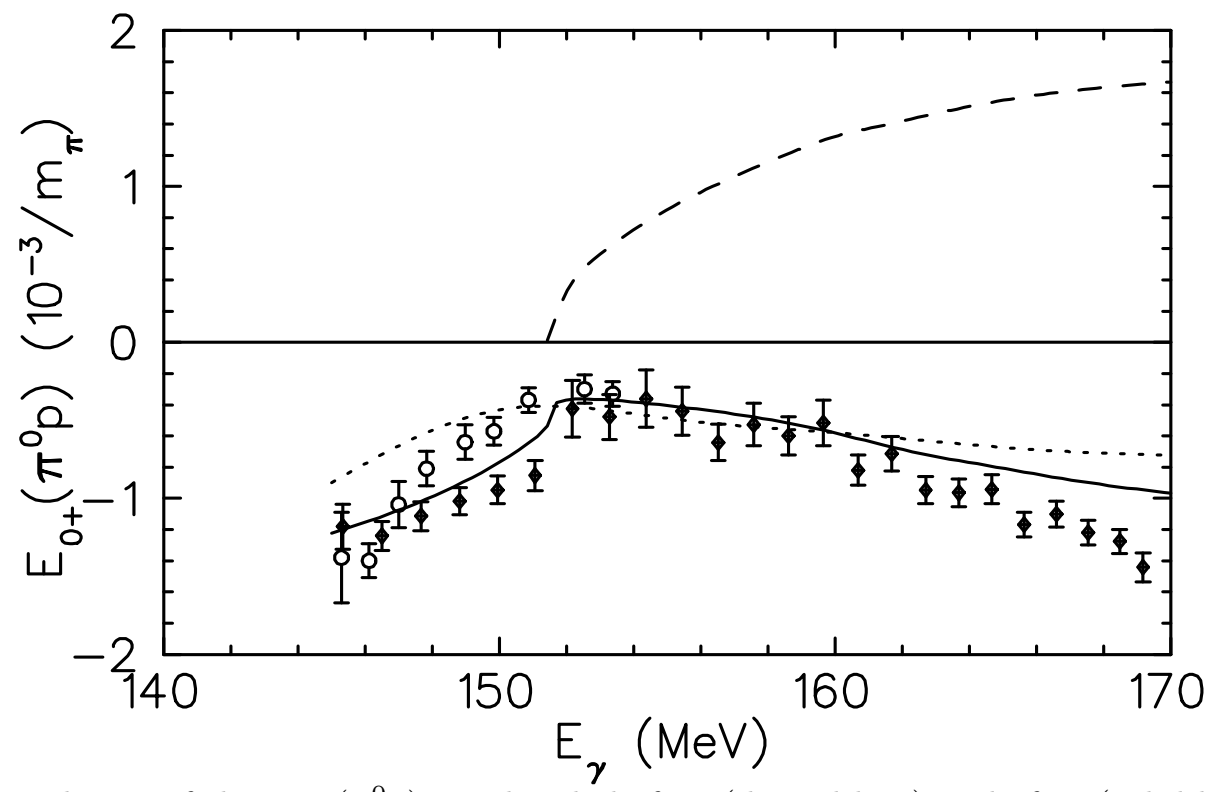

FIG. 2. Real part of the $E_{0+}\left(\pi^{0} p\right)$ amplitude before (dotted line) and after (solid line) iteration of Eqs. (11). The dashed line is the imaginary part of this amplitude. Data from Ref. [7] (circles) and Ref. [8] (diamonds). 

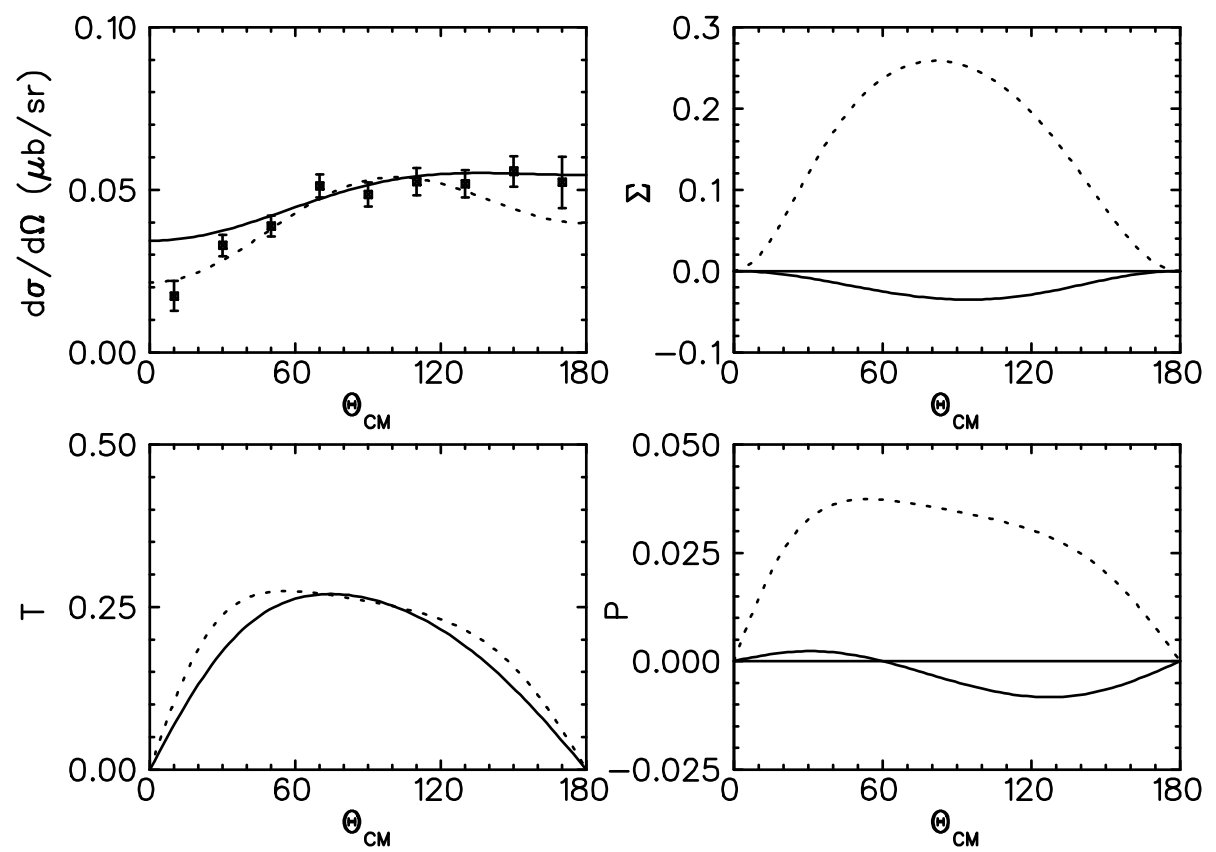

FIG. 3. Differential cross section and single polarization observables for the reaction $\gamma p \rightarrow \pi^{0} p$ at $E_{\gamma}=151.69 \mathrm{MeV}$ (solid lines). The dotted lines show our result after reducing the $M_{1-}$ amplitude by 50\%. Experimental data from Ref. [0].
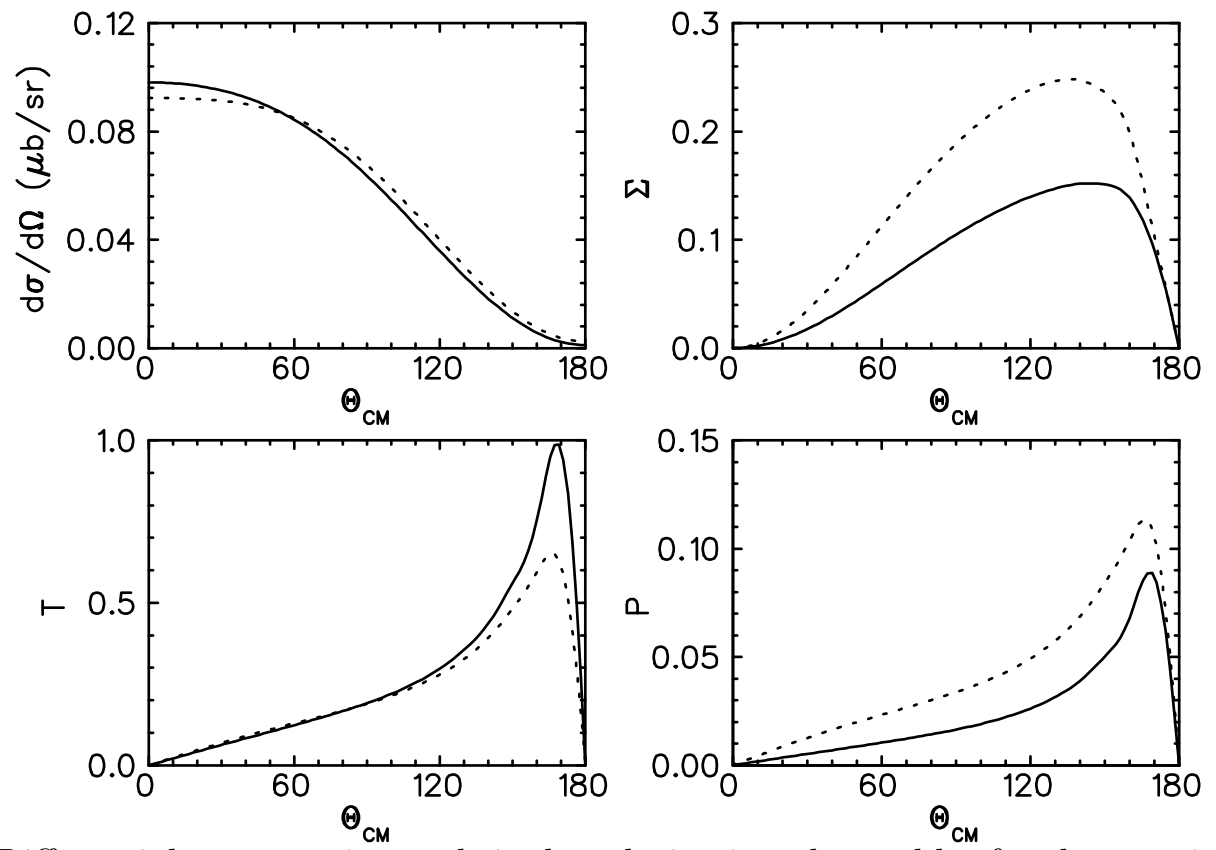

FIG. 4. Differential cross section and single polarization observables for the reaction $\gamma n \rightarrow \pi^{0} n$ at $E_{\gamma}=151.69 \mathrm{MeV}$ (solid lines). The dotted lines show our result after reducing the $M_{1-}$ amplitude by $50 \%$. 